\title{
VARIA
}

\section{A PROPÓSITO DE LOS DIBUJOS INÉDITOS DEL INSTITUTO VALENCIA DE DON JUAN ${ }^{1}$}

El Instituto Valencia de Don Juan ${ }^{2}$, constituido notarialmente en 1916 conforme a las ideas de Guillermo Joaquín de Osma y Scull (La Habana, 1853-Madrid, 1922), casado con la última condesa de dicho título, Adelaida Crooke y Guzmán (Madrid, 1863-París, 1918) ${ }^{3}$, guarda, en un reformado palacete de la calle Fortuny de Madrid, importantes colecciones de pinturas, esculturas, tapices, bordados, armas, azulejos, loza, alfarería, azabaches, monedas, muebles, libros y documentos, que el matrimonio logró reunir, provenientes de sus antepasados y adquiridos por ellos. Entre tan valiosos fondos casi pasan desapercibidos los veintiocho dibujos, que hemos catalogado recientemente, los cuales no pueden considerarse una colección sino un conjunto de piezas que llegaron aleatoriamente a la Institución, la mayoría de procedencia hoy desconocida, realizados entre principios de los siglos XVI y Xx. De este grupo de rasguños ya han salido a la luz los que, posiblemente, son más destacados, realizados por artistas de primera fila ${ }^{4}$, pero aún quedan ejemplares significativos, dignos de estudio, de los que damos cuen-

\footnotetext{
1 Deseo agradecer al Profesor D. Alfonso Emilio Pérez Sánchez las puntualizaciones que, amablemente, me ha proporcionado para este artículo acabado en diciembre de 1999.

2 Andrés, G. de, La fundación del Instituto y Museo Valencia de Don Juan, Madrid, 1984, pp. 5-32.

${ }^{3}$ Fruto de la actividad de Adelaida Crooke es un diario que escribió en su viaje a Egipto y Tierra Santa (comienza el 12-4-1897 y termina el 17-7-1897), escrito a lápiz $(22 \times 17 \mathrm{cms})$, acompañando a la ex Emperatriz Eugenia de Montijo y la Duquesa de Alba, que se conserva en el Instituto. Adelaida Crooke colaboró con su marido en el inventario de la importante colección de cerámica y en alguna ficha consta algún esquema que podría haber realizado. No sería extraño que la Condesa también dibujase y en el Instituto se conservan algunas acuarelas de flores, sin firmar, que podrían ser de su mano. Entre la documentación de la biblioteca localizamos una ficha que reza: «Dibujos a pluma de Venecia y Verona por Adelita en el portalón del Salón», ejemplares que no se incorporan a este inventario al no aparecer entre los fondos consultados, que podrían tener alguna relación con esta actividad aunque los nombres no coinciden.

${ }^{4}$ Esquematizamos los datos de los dibujos ya editados, seleccionando, de las existentes, una publicación para cada uno: Planta de la Catedral de Granada, ca. 1594, lápiz repasado con pluma a tinta sepia, de Juan de la Vega, n. ${ }^{\circ}$ inv. ${ }^{\circ}$ Envío 8 , 2. " parte, caja 13 (Rodríguez Ruiz, Delfín, Un dibujo inédito de la planta de la Catedral de Granada, «A. E. A», n . 280, Madrid, 1997, págs. 335-374), está previsto que este dibujo figure en la exposición «Carlos V, las armas y las letras» en el Hospital Real de Granada, organizada por la Sociedad Estatal para la Conmemoración de los centenarios de Felipe II y Carlos V, comisariadas por Fernando Marías y Felipe Pereda, que se inaugurará en abril de 2000; Los tres ángeles, lápiz carbón sobre papel verjurado, de Navarrete «el Mudo», n..$^{\circ}$ inv. ${ }^{\circ}$ I-6077 (Mulcahy, R., Juan F. Navarrete, pintor de Felipe II, Madrid, 1999, p. 47, fig. 18); San Lucas pintando a la Virgen, lápiz negro, pluma y aguada sepia con toques de blanco sobre papel verjurado, de V. Carducho, n. ${ }^{\circ}$ inv. ${ }^{\circ}$ I-7863 (Angulo, D. y Pérez Sánchez, A. E., A corpus of Spanish drawings, Madrid 1600-1650, II, London, 1977, fig. 194); Angeles volando, pluma y aguada sombra tostada sobre papel verjurado, de Alonso Cano, n. ${ }^{\circ}$ inv. ${ }^{\circ}$ I-6086 (Sánchez Cantón, F.J, Dibujos españoles, Madrid, 1930, IV, CCCXXXIV); Virgen con S. Felipe y S. Francisco, el dibujo más espectacular y más conocido de esta colección, de Francisco Rizi, lápiz, pluma y aguada sombra natural sobre papel verjurado, n..$^{\circ}$ inv. ${ }^{\circ}$ I-6080 (Pérez Sánchez, A. E., El dibujo europeo en tiempos de Velázquez, Madrid, 1999); Cabezas de niños, de Antonio del Castillo, sanguina sobre papel verjurado, n..$^{\circ}$ inv. ${ }^{\circ}$ I-6087 (Muller, P., The
} 



Fig. 1. Anónimo «Traza parcial de la Catedral de Granada».

Fig. 2. Planta de la Catedral de Granada. (Camón Aznar, Summa Artis, tomo XVII).

Figs. 3 y 4. Plantas de la Catedral de Salamanca (Chueca, Ars Hispaniae, tomo XI). 


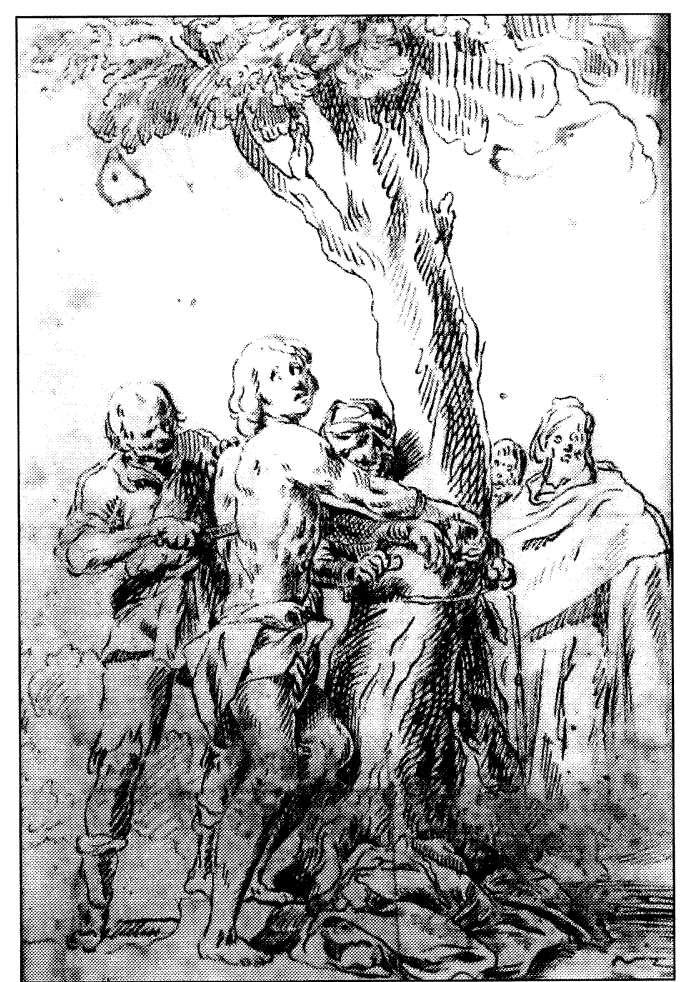

5

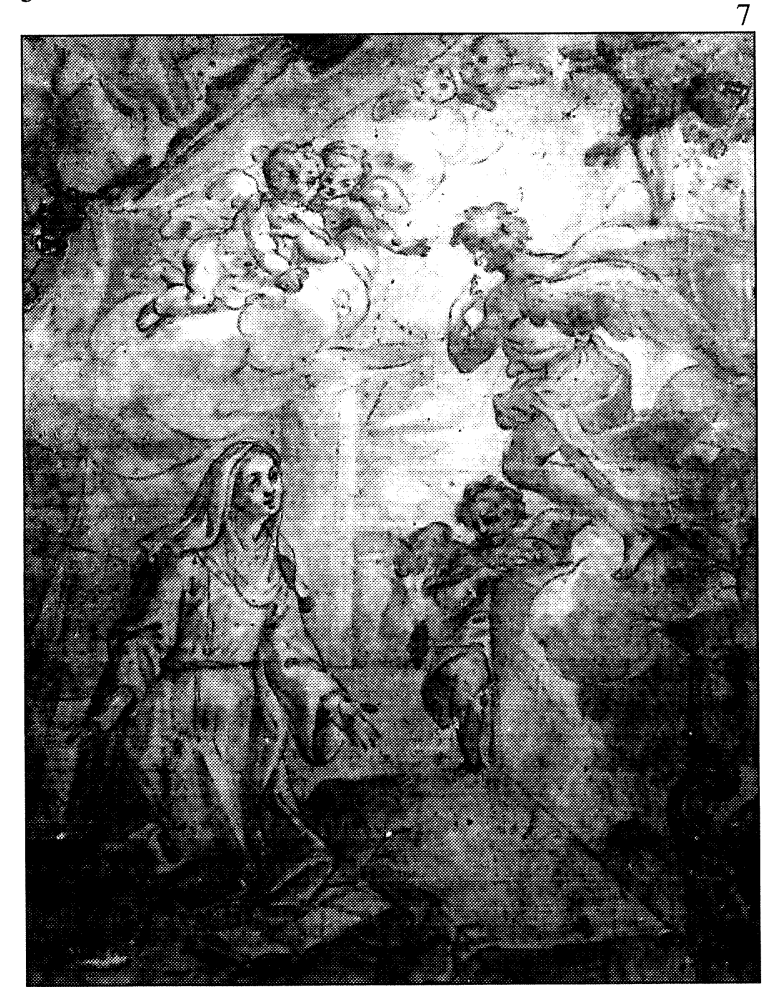

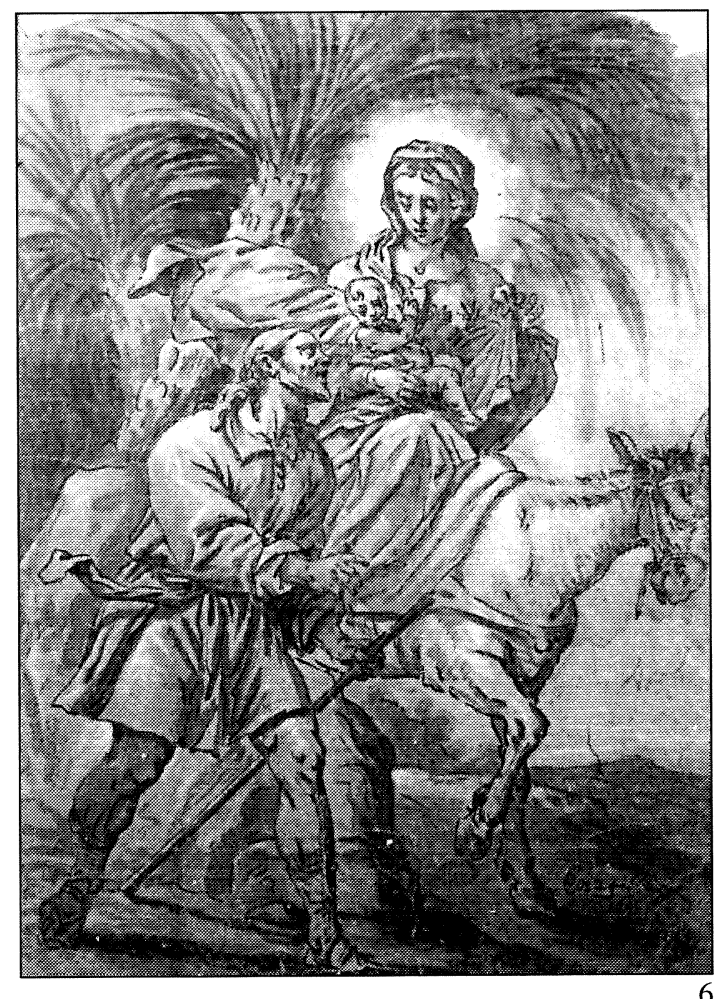

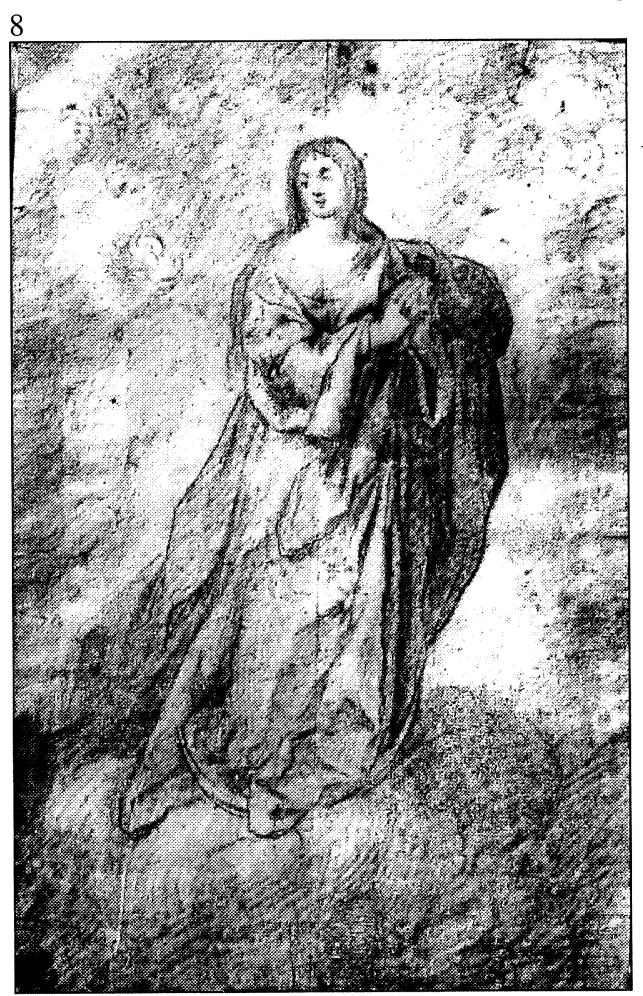

Fig. 5. Antonio del Castillo Saavedra, Martirio de San Zoilo.

Fig. 6. Juan Conchillos Falcó, Huida a Egipto.

Fig. 7. Anónimo, Anunciación.

Fig. 8. Anónimo, Inmaculada Concepción. 
ta a renglón seguido, con la modesta pretensión de ponerlos a disposición de la comunidad científica.

Entre las obras del XVII sobresale el Martirio de San Zoilo ${ }^{5}$, de Antonio del Castillo Saavedra (Córdoba, 1616-1668) ${ }^{6}$, miembro de una familia de pintores activos en Andalucía en el referido siglo, el mejor representante de la escuela cordobesa de su tiempo, dibujante prolífico y hábil, cuyas creaciones a tinta, utilizando la pluma de caña gruesa a la manera de Herrera, el carbón o la aguada, se refieren a temas religiosos, como el presente, mitológicos, decorativos, y abundantes estudios de cabezas que podrían ser un intento de cartilla de dibujo ${ }^{7}$, en los que se comprueba fuerte influencia de los grabados realizados a partir de diseños de Abraham Bloemaert (1566-1651), cuyas estampas, al igual que sucedió con las de otros europeos, invadieron la España Barroca y se usaron como modelo para sus creaciones por artistas del XVII ${ }^{8}$.

Del valenciano Juan Conchillos Falcó (1641-1711) ${ }^{9}$, discípulo de Esteban March y formado en Madrid, donde contactó con su paisano García Hidalgo, el autor de una famosa cartilla de dibujo ${ }^{10}$, sabemos que dejó abundantes papeles, muchos ejercicios de academias para las que gustaba hacer uso del carbón con clarión, de las que, según Palomino, no dejaba noche sin trazar una ${ }^{11}$. También que cultivó representaciones de angelotes y asuntos religiosos, como el del Instituto, referido a la Huida a Egipto, resuelto a la aguada con acusados contornos, cuidadosa-

drawings of A. del Castillo, Tesis, N. York University, 1963, fig. 88). De Francisco de Goya hay cuatro dibujos realizados a lápiz y clarión, dos son unos Majos, $\mathrm{n}^{\circ \mathrm{s}}$ inv. ${ }^{\circ}$ I-6088 r. ${ }^{\circ}$ e I-6089 (VV. AA., Permanencia de la memoria. Cartones para tapices y dibujos de Goya, Zaragoza, 1997, pp. 133 y 134); dos representan la Entrega de llaves de Sevilla a S. Fernando, n. ${ }^{\circ}$ inv. $^{\circ}$ : I-6088 v. ${ }^{\circ}$ e I-6085 (Gassier, P. y Wilson, J., Vie et oeuvre de F. de Goya, Fribourg, 1970, p. 196 y Gassier, P., Les

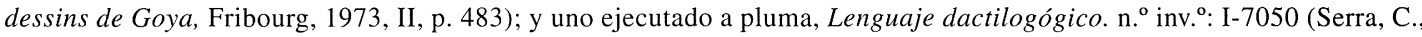
Una lámina de Goya ....lenguaje para sordos, «El País», Madrid, 23-VI-97, p. 36), del que existe un antecedente español en la obra de Juan Pablo Bonet, Reduction de las letras y arte para enseñar a hablar los mudos, Francisco Abarca de Angulo, Madrid, 1620 (BNM R-8155) y otro en algunas láminas de la cartilla de García Hidalgo, J., Principios para estudiar el nobilísimo y real arte de la pintura, s. i., (1693), Madrid, 1965. Por último un dibujo a lápiz tricolor, Retrato de canónigo, de Vicente López, n. o inv. I-6090 (López, J. L., Vicente López, Madrid, 1999, II, lám. 259).

${ }^{5} \mathrm{~N} .^{\circ}$ inv. ${ }^{\circ} \mathrm{I}-6084,270 \times 185 \mathrm{~mm}$, pluma gruesa a tinta sombra tostada sobre papel verjurado y toques de blanco. En a.i.d. «Castillo». Presenta dobleces y pérdidas de superficie por acción biológica en parte central inferior.

${ }^{6}$ Sobre Antonio del Castillo, además del pionero estudio de Priscila Muller (The drawings of Antonio del Castillo y Saavedra, New York University, 1963), véanse los trabajos más recientes: García de la Torre, F., Dibujos del Museo de Bellas Artes de Córdoba, Sevilla, 1997, pp. 91-121; Nancarrov Taggard, M., Antonio del Castillo en los Museos Valencianos, «A.E.A.», nºs 259-260, Madrid, 1992, pp. 322-334; Pérez Sánchez, A. E., El dibujo español en los siglos de Oro, Madrid, 1980, pp. 57-95, láms. 84-95; Idem, Historia del dibujo en España, Madrid, 1986, pp. 183-186; Idem, Maîtres des XVI et XVII ${ }^{e}$ siècles, Louvre, Musées Nationaux, París, 1991, pp. 227-229; Valverde Candil, M., «Los dibujos de Antonio del Castillo», en VV. AA, Antonio del Castillo y su época, Diputación Provincial, Córdoba, 1986, pp. 105-116.

${ }^{7}$ La hipotética existencia de una cartilla de dibujo de Antonio del Castillo, referida a estudios de cabezas humanas, en cuya reconstrucción llevamos trabajando hace tiempo, ha sido apuntada, entre otros, por Pérez Sánchez (Historia del dibujo en España, Madrid, 1986, págs. 183-186).

${ }^{8}$ La utilidad de la estampa como modelo para los artistas es asunto que ya ocupó a importantes tratadistas del barroco español como Francisco Pacheco (Arte de la pintura, 1649, ed. Sánchez Cantón, Madrid, 1956, p. 79), Jusepe Martínez (Discursos practicables del nobilísimo arte de la pintura, ca. 1675, ed. V. Carderera, Madrid, 1866, pp. 5, 22 y 139) y Antonio Palomino (Museo pictórico, 1715, ed. Ceán Bermúdez, Madrid, 1947, pp. 522, 523, 533 y 534). Los estudios sobre las correspondencias entre estampas y obras concretas de artistas, ejemplos de haber sido utilizadas como referente iconográfico, es tema que se recoge recientemente por los historiadores pudiendo destacarse algunos estudios como los de: Bonet Correa, A., «El grabado en España y su significación», en VV. AA, Estampas. Cinco siglos de imagen impresa, Madrid, 1982, pp. 9-12; Carrete Parrondo, J., El grabado y la estampa barroca, «Summa Artis», vol. XXXI, Madrid, 1987, pp. 340342; Navarrete Prieto, B., La pintura andaluza del siglo xvit y sus fuentes grabadas, Madrid, 1998; Pérez Sánchez, A. E, «La estampa como modelo», en VV. AA., El dibujo, razón, orden y artificio, Zaragoza, 1992, pp. 77-99; Idem, De pintura y pintores. La configuración de los modelos visuales en la pintura española, Madrid, 1993.

9 Sobre dibujos de Juan Conchillos véanse: Pérez Sánchez, A. E., El dibujo español de los siglos de Oro, op. cit, pp. 66-68 y láms. 110-117; Angulo, D., y Pérez Sánchez, A. E., A Corpus of Spanish Drawings, Valencia, 1600-1700, IV, London, 1988, pp. 19-28, lams. 1-76; Espinos Díaz, A., Dibujos de los siglos xvi y xvir en el Museo de San Carlos de Valencia, «Archivo de Arte Valenciano», Valencia, 1974, pp. 61-68 y 1975, pp. 72-84; Idem, Dibujos valencianos del s. XVII, Sevilla, 1997, pp. 210-277.

10 García Hidalgo, J., Principios para estudiar el nobilísimo y real arte de la pintura, con todo..., ca. 1693, Madrid, s. i, ed. de Rodríguez Moñino, Madrid, 1965.

11 Palomino, A., El Parnaso pintoresco y laureado, 1724, ed. Ceán Bermúdez, Madrid, 1947, pág. 1133. 



Fig. 9. Anónimo, Venus y Cupido

Fig 10. Bloemaert, Venus y Cupido.Estampa grabada por Boëtius Adams Bolswert, ca. 1610

Fig. 11. Luis de Madrazo y Kuntz, Desnudo femenino con perro.

Fig. 12. Joaquín Araujo Ruano, Aldeana sentada. 


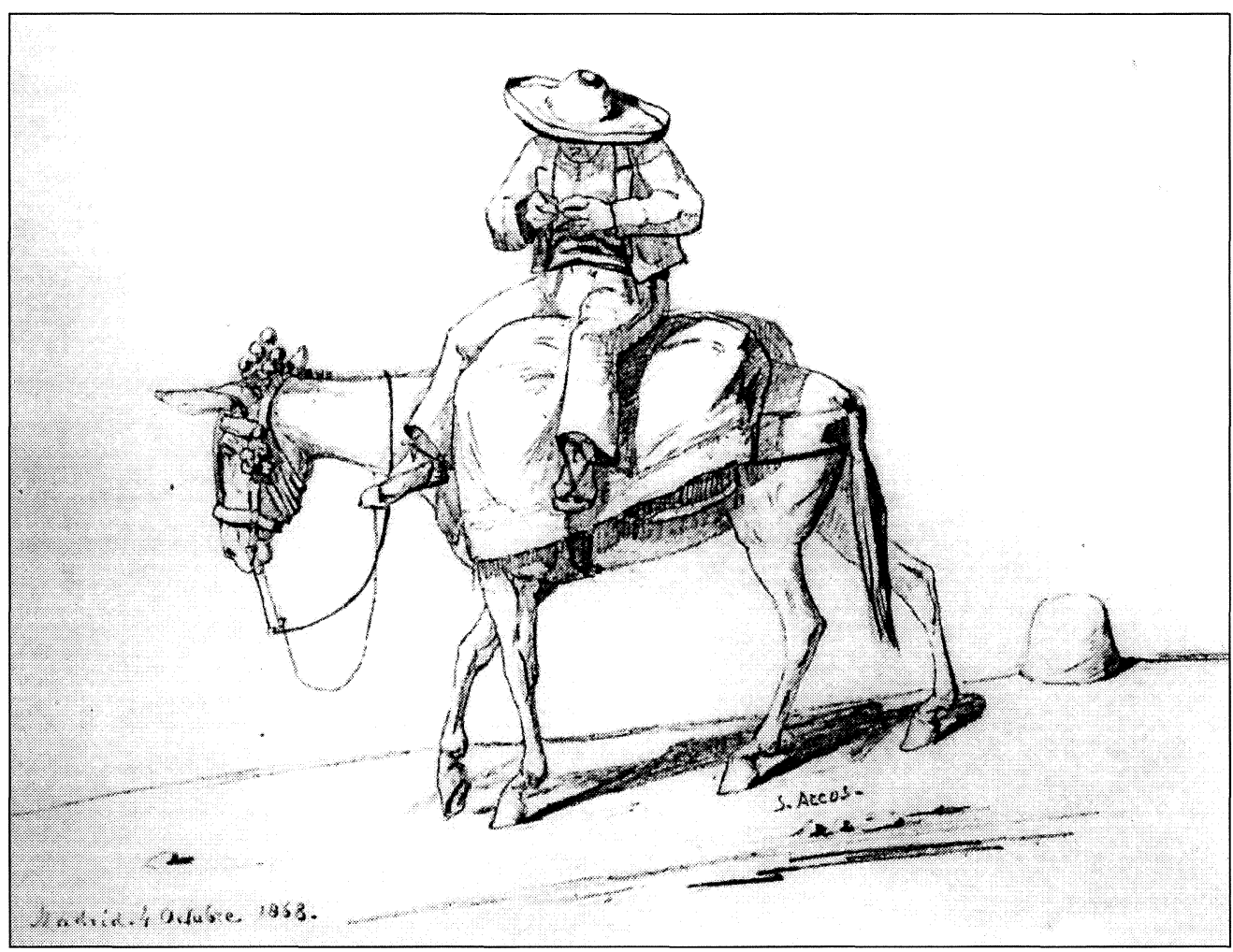

13
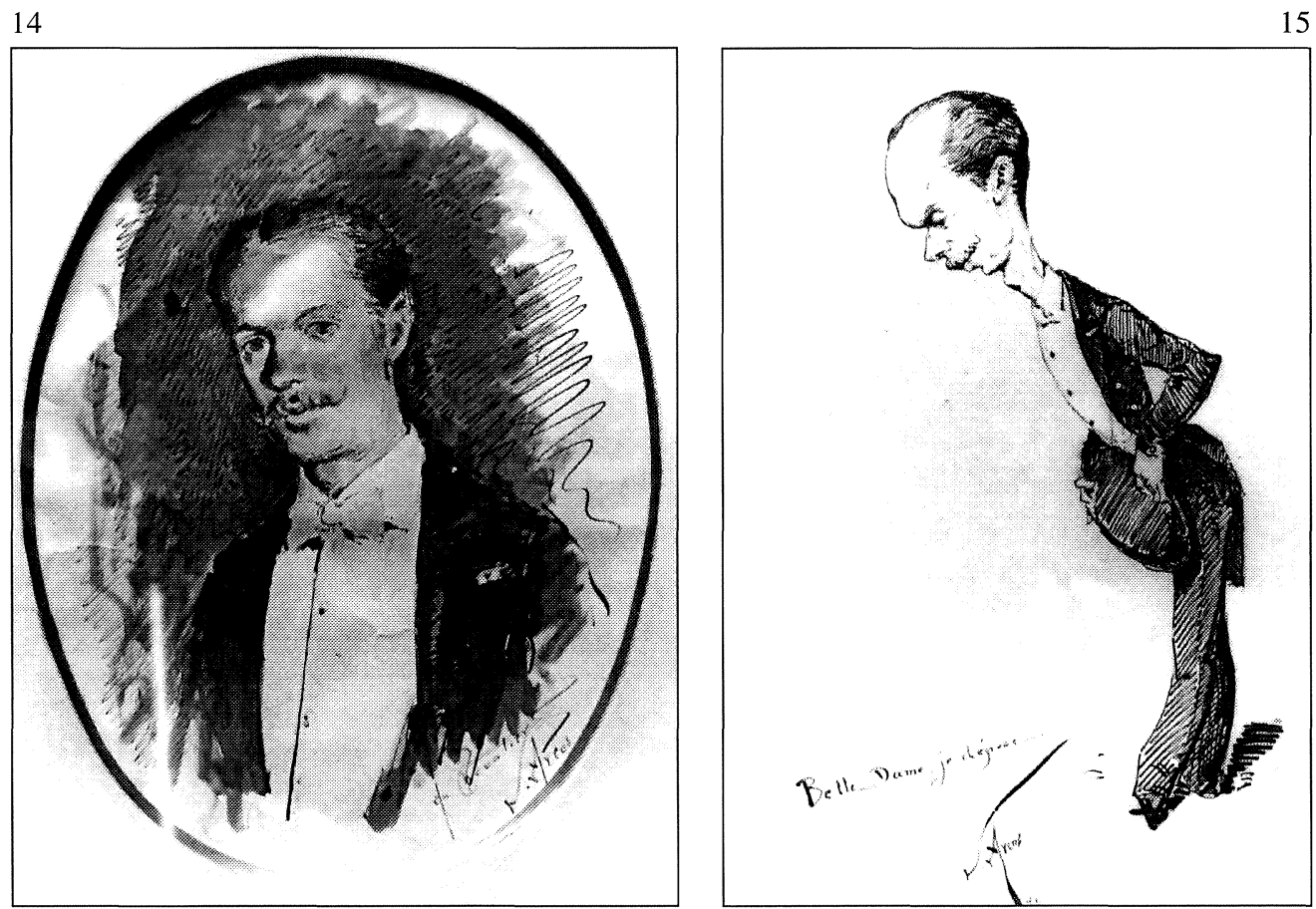

Fig. 13. Santiago Arcos y Megalde, Hombre a caballo.

Fig. 14. Santiago Arcos y Megalde, Retrato de Guillermo de Osma.

Fig. 15. Santiago Arcos y Megalde, Caricatura de Guillermo de Osma. 
mente fechado, como solía hacer, el 24 de mayo de $1695^{12}$. Completa el siglo Xvil una aguada sepia anaranjada, que representa la Anunciación, de autor anónimo, en la que se intuyen deudas canescas, que esconde el previo encajado delineado a lápiz, adquiriendo un agradable aspecto que no logra ocultar cierta rudeza e impericia en la resolución de algunas figuras ${ }^{13}$.

Posiblemente de principios del XVIII es una Inmaculada, realizada a sanguina, atribuida, según nota antigua que consta al pie del propio dibujo, erróneamente a Murillo. La falta de seguridad y corrección en el trazo de este dibujo, bien distinta de la que exhibe el maestro sevillano en sus papeles, hacen imposible imputarle esta obra que más parece ser ejercicio de pintor dieciochesco aún no formado o de segunda fila ${ }^{14}$.

De 1732 es un atrayente ejercicio a la aguada gris, Venus y Cupido ${ }^{15}$, de autor anónimo, que hemos identificado como copia exacta de un grabado del mismo título abierto por Boëtius Adams Bolswert (1580-1633), a partir de un dibujo del, antes citado, holandés Abraham Bloemaert, lo que prueba que sus estampas no sólo se usaron como modelo iconográfico en el Xvil, como antes hemos mencionado, sino que los artistas las siguieron utilizando en la siguiente centuria ${ }^{16}$.

El resto de las piezas datan del siglo xIx y principios del xx entre las que aparece un sugerente Desnudo femenino con perro, de 1845, de Luis de Madrazo y Kuntz (Madrid, 18251897), hermano e hijo de pintores, que se instruyó en el clasicismo dominante, sucesivamente, en el estudio de su padre, en la Escuela de B. A. de San Fernando y luego en Italia donde vivió la influencia del nazareno Overbek, desarrollando una obra de representaciones bíblicas, religiosas y abundantes retratos ${ }^{17}$. El dibujo, un tanto relamido e incorrecto en el entronque del brazo derecho al cuerpo, lo que es entendible en una obra de juventud como el presente, bastante acabado, que resuelve con ligera aguada, definiendo los contornos, a lápiz y perfilando las formas mientras que para el sombreado se sirve del habitual ejercicio de cruce de líneas a la manera de los grabadores de planchas ${ }^{18}$.

También está representado Joaquín Araujo Ruano (C. Real, 1851-1894) ${ }^{19}$, formado en la Academia de Bellas Artes de San Fernando, discípulo de Suárez Llanos y después en París con Leon Bonnat. Fue pintor de lienzos de asuntos populares y costumbristas, cultivando el aguafuerte, cuyos trabajos fueron reconocidos especialmente en Londres. Dejó muchos dibujos, de los que varios se guardan en el Prado y en la Real Academia de Bellas Artes de San Fernando, entre cuyos motivos constan estudios de muchachos, desnudos o aldeanas como el ejemplar de

${ }^{12} \mathrm{~N}^{\circ}$ inv. $^{\circ} \mathrm{I}-6079,340 \times 240 \mathrm{~mm}$, pluma y aguada sombra natural, con toques de blanco, sobre papel ligeramente coloreado de natural. En a.i.d. «En 24 de Mayo 1695 Conchillos». En reverso: $1 .^{\circ} . \mathrm{R}^{\mathrm{s}}$.

$13 \mathrm{~N} .^{\circ}$ inv. $^{\circ} \mathrm{I}-7864,220 \times 170 \mathrm{~mm}$, lápiz y aguada sepia sobre papel verjurado. En a.i.i. «2» a tinta. Muestra dobleces y pérdidas de papel, toscamente restaurado de antiguo con parches.

${ }^{14} \mathrm{~N} .^{\circ}$ inv. $^{\circ} \mathrm{I}-6082,278 \times 180 \mathrm{~mm}$, sanguina sobre papel verjurado. En a.i.i., a lápiz, «Murillo». Tiene manchas y presenta arrugas de haber estado doblado por la mitad.

${ }^{15} \mathrm{~N}$. $^{\circ}$ inv. $^{\circ} \mathrm{I}-6078,185 \times 142 \mathrm{~mm}$, pluma tinta sepia y aguada grisácea sobre papel verjurado. En a.i.i. «1732» . En reverso, en a.s.i. «en $1 / 5 »$ y lo que parece una rúbrica.

${ }^{16}$ Para los grabados de Bloemaert véase el amplísimo estudio, con destacado aparato gráfico, de Roethlisberger, M. G., Abraham Bloemaert and his sons, Paintings ad Prints, Belgium, 1993, 2 vols.

17 Sobre los Madrazo véase: Madrazo López de Calle, M. de, Federico de Madrazo, II vols. Monografías de Arte «Estrella», Madrid, 1920-1921; Pantorba, B. de, Los Madrazos, ed. Iberia, Barcelona, 1947; González López, C, Federico de Madrazo y Kuntz, Barcelona, 1981; Puente, J. de la y San Nicolás, J, Madrazo (F.), «Los Genios de la Pintura Española», Sarpe, Madrid, 1988; Gállego, Julián y otros, Los Madrazo, una familia de artistas. Exp. Museo Municipal de Madrid, 1988; Gaya Nuño, Juan, El Arte del siglo XIX. "Ars Hispaniae», XIX, Madrid, 1966, pp. 246 y ss.; Gómez Moreno, M. E., Pintura y escultura españolas del s. XIX, «Summa Artis», vol. XXXV, Madrid, 1994, pp. 313-314; y VV.AA, Cien años de pintura en España y Portugal, 1830-1930, Anticuaria, Madrid, 1988-1992, vol. V, pp. 116-122.

${ }^{18} \mathrm{~N}^{\circ}$ inv. $^{\circ}$ I-7842, $161 \times 136 \mathrm{~mm}$. Aguada sepia y lápiz negro sobre papel amarillento. En a.i.d. «L. de Madrazo, 1845».

19 Véanse: González Ortiz, J., Joaquín Araujo y Ruano (1851-1894), pintor y aguafuertista. Extra. Feria de Ciudad Real, «Periódico Lanza». Agosto, 1986; Osorio y Bernard, M., Galería Biográfica de Artistas españoles del siglo xIX, Imprenta Moreno y Rojas, Madrid, 1883-1884, p. 47; Pantorba, B. de, Historia y crítica de las exposiciones nacionales de Bellas Artes, Jesús Ramón G. ${ }^{a}$ Rama, Madrid, 1980, p. 369; VV.AA., Cien años de pintura en España y Portugal, 1830-1930, Anticuaria, op. cit. vol. I, p. 205. 


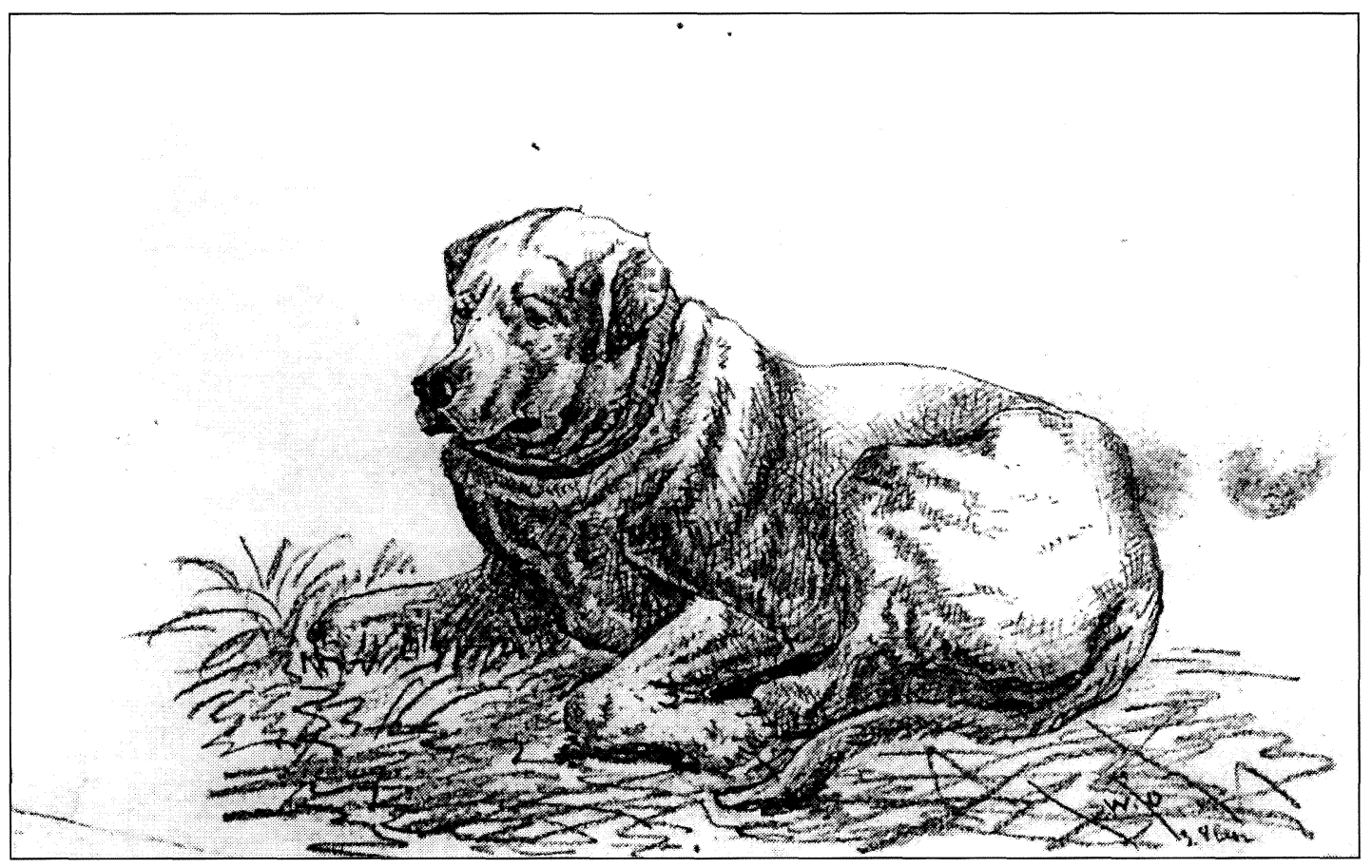

16
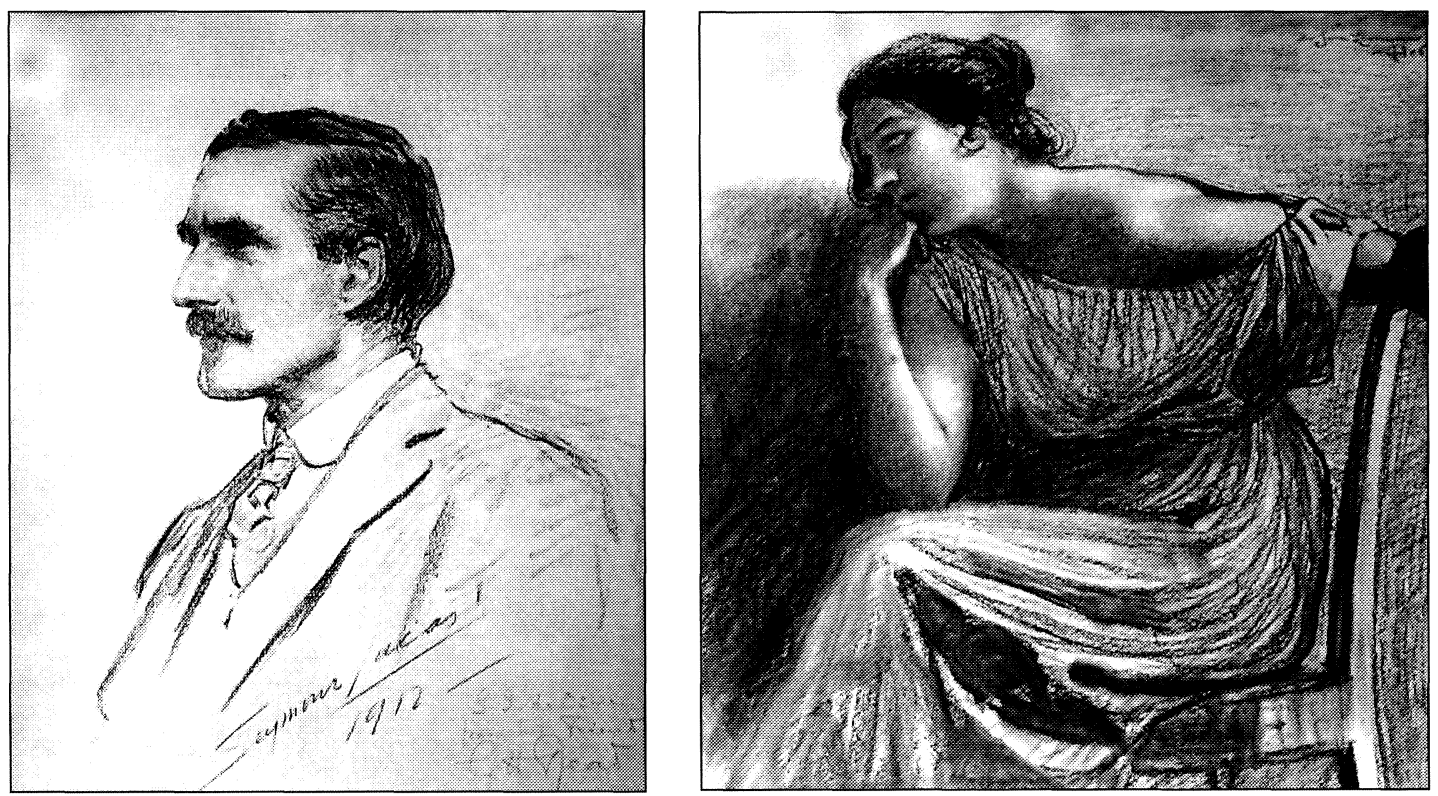

Fig. 16. Guillermo Joaquín de Osma, Perro recostado.

Fig. 17. John Seymour Lucas, Retrato de Charles H. Read.

Fig. 18. Juan Llimona Bruguera, Mujer sentada pensativa. 
la Figura sentada, realizado a pluma y aguada, del Instituto Valencia de Don Juan, que está firmado por el propio autor y fechado en $1880^{20}$.

Tres son los ejemplares del chileno Santiago Arcos Megalde (Santiago de Chile, s. XIX-S. Sebastián, 1915) ${ }^{21}$ afincado en Madrid, que fue premiado en exposiciones de Madrid (1878) y París (1900), cultivador de la pintura de género histórico, mitológico y costumbrista: un Hombre a caballo, a lápiz de pulcro perfilado ${ }^{22}$, un fresco y ágil Retrato de G. De Osma, a la agua$\mathrm{da}^{23}$ y una caricatura del mismo personaje de $1879^{24}$.

Atractivo es un dibujo a lápiz carbón y sanguina, de 1912, dedicado por el retratado, Charles H. Read, que fue conservador del Museo Británico, a G. De Osma, con quien mantuvo relación intelectual, cuya ejecución corrẹsponde al pintor británico John Seymour Lucas (Londres, 1849-1923) ${ }^{25}$, pintor de asuntos de historia, escenas de género y retratos además de acuarelista, el cual hizo viaje de estudio a España y desarrolló su obra en Londres donde expuso regularmente desde $1871^{26}$.

Precisamente, como muestra de la actividad multidisciplinar del culto Guillermo de Osma ${ }^{27}$, el creador del Instituto de Valencia de Don Juan, «Willy» para sus amigos de tertulias ${ }^{28}$, economista, coleccionista, diplomático y político, hemos encontrado un dibujo a lápiz, de su mano, que representa un Perro recostado, el cual es preparatorio de un óleo que también pintó el polifacético personaje que da nombre al Instituto ${ }^{29}$.

Asimismo, consta una representativa obra de la escuela catalana de principios del siglo xx realizada por Juan Llimona Bruguera (Barcelona, 1860-1926) ${ }^{30}$, pintor que tuvo sus inicios con Martí y Alsina y Antonio Caba en la escuela de la Lonja, instruido después en Italia a donde marchó con su hermano, el escultor, José (1864-1934), formó parte del llamado «Cercle de San Lluc» de orientación católica, fundado en 1893 e hizo abundante pintura religiosa, al-

${ }^{20} \mathrm{~N} .^{\circ}$ inv. $^{\circ}$ I-7841, 310 x $215 \mathrm{~mm}$, pluma y aguada sombra tostada sobre papel «Montanel». En anverso, a.i.i. «26 de ocbre 80 J. Araujo» y en a. i. d. sello de «TESTAMENTARIA DE J. ARAUJO» y en el reverso «Joaquín Araujo Ruano 1851-1894».

${ }_{21}$ Osorio y Bernard, M., Galería Bibliográfica de Artistas españoles del siglo XIX, Imprenta Moreno y Rojas, Madrid, 1883-1884, p. 47; Pantorba, B. de, Historia y crítica de las exposiciones nacionales de Bellas Artes, Madrid, 1980, p. 369; Beruete y Moret, A. de, Historia de la pintura española en el siglo XIX, elementos nacionales y extranjeros que han influido en ella. Ruiz Hermanos, Madrid, 1926, p. 124; VV.AA, Cien años de pintura en España y Portugal, 1830-1930, Anticuaria, op. cit. Vol. I, pp. 207-208.

${ }^{22} \mathrm{~N}^{\circ}{ }^{\text {inv. }}{ }^{\circ} \mathrm{I}-7843,197 \times 242 \mathrm{~mm}$, lápiz negro sobre papel. En a.i.i. «Madrid, 4 Octubre $1868 »$ y en el derecho «S. Arcos».

${ }^{23} \mathrm{~N}^{\circ}$ inv. $^{\circ} \mathrm{I}-6197,270 \times 210 \mathrm{~mm}$, tinta y aguada sepia sobre papel amarillento. En a.i.d. «P/u Chevalier S. Arcos»

${ }^{24} \mathrm{~N}^{\circ}$ inv. $^{\circ} \mathrm{I}-6196,340 \times 235 \mathrm{~mm}$, tinta sobre papel amarillento. En a.i. i. «Belle Dame, je d' epose ... S. Arcos».

${ }_{25}$ Benezit, E., Dictionnaire des peintres, sculpteurs, dessinateurs et graveurs, Grund, 1999, tomo. VIII.

${ }^{26}$ N. ${ }^{\circ}$ inv. ${ }^{\circ} . \mathrm{I}-6214,255$ x 310 mm, lápiz carbón y sanguina sobre cartón. Al pie: «Seymour Lucas. 1912». En a.i.d. «G.J. de Osma fron his Friend C.H. Read».

${ }_{27}$ Sobre el primer director del Instituto Valencia de Don Juan, se han escrito dos recientes estudios: Barrio Moya, José Luis, Un coleccionista atípico: Don Guillermo Joaquín de Osma, «Goya», 276, Madrid, 1998, pp. 163-374; y el de M. ${ }^{\text { }}$ Jesús González, «Guillermo de Osma o los avatares de un proteccionista preocupado», en VV.AA., Los Ministros de Hacienda en el período de la Restauración, Prensa Universitaria, Zaragoza, 2000 (en prensa).

${ }_{28}$ Respecto a las tertulias culturales «masculinas», que se realizaban en el Instituto entre 1896 y 1904 , en las que se distingue la presencia de personajes como el Duque de Alba, Tormo, Gómez Moreno, Sánchez Cantón, etc, queda un curioso álbum de retratos-caricaturas de José María Florit (Recuerdo de la Tertulia dominguera del Conde de Valencia de Don Juan: 1896-1904. Arqueólogos, anticuarios y Bibliófilos más o menos chiflados, Fototipia de Hauser y Menet, Madrid, 1904).

${ }^{29} \mathrm{~N}^{\circ}$ inv. $^{\circ} \mathrm{I}-7868,135 \times 210 \mathrm{~mm}$, lápiz negro sobre papel. En anverso «W.O» y «3.8 leon» y al reverso «30 octubre». El dibujo tiene su réplica en un pequeño óleo/lienzo, también de G. de Osma, que guarda el Instituto (n. ${ }^{\circ}$ inv. ${ }^{\circ}$ I-7054) el cual está en proceso de restauración actualmente.

${ }^{30}$ Osorio y Bernard, M., Galería Bibliográfica de Artistas españoles del s. XIX, op. cit, p. 375, en donde equivoca el nombre de Juan por el de su hermano José; Pantorba, B. de, Historia y crítica de las exposiciones nacionales de Bellas Artes, Madrid, 1980, op. cit, p. 430; Rucabado, R., L'Art dels germans Llimona, «Vell i Nou», Barcelona, agosto, 1920; Llimona, Josep, Josep Llimona y Joan Llimona: vida y obra, Nuevo Arte, Thor, Barcelona, 1977, donde se recoge amplia bibliografía; VV. AA, Cien años de pintura en España y Portugal, 1830-1930, Anticuaria, vol. V, 33; Bozal, Valeriano, Pintura y escultura españolas del siglo xx, 1900-1939, «Summa Artis», Madrid, 1992, pp. 18-19 y 32-35; Triadó, J. R., La pintura catalana. Los protagonistas de los ss. XIX y XX, Skira. Carrogio, Barcelona, 1994, pp. 33-34. 


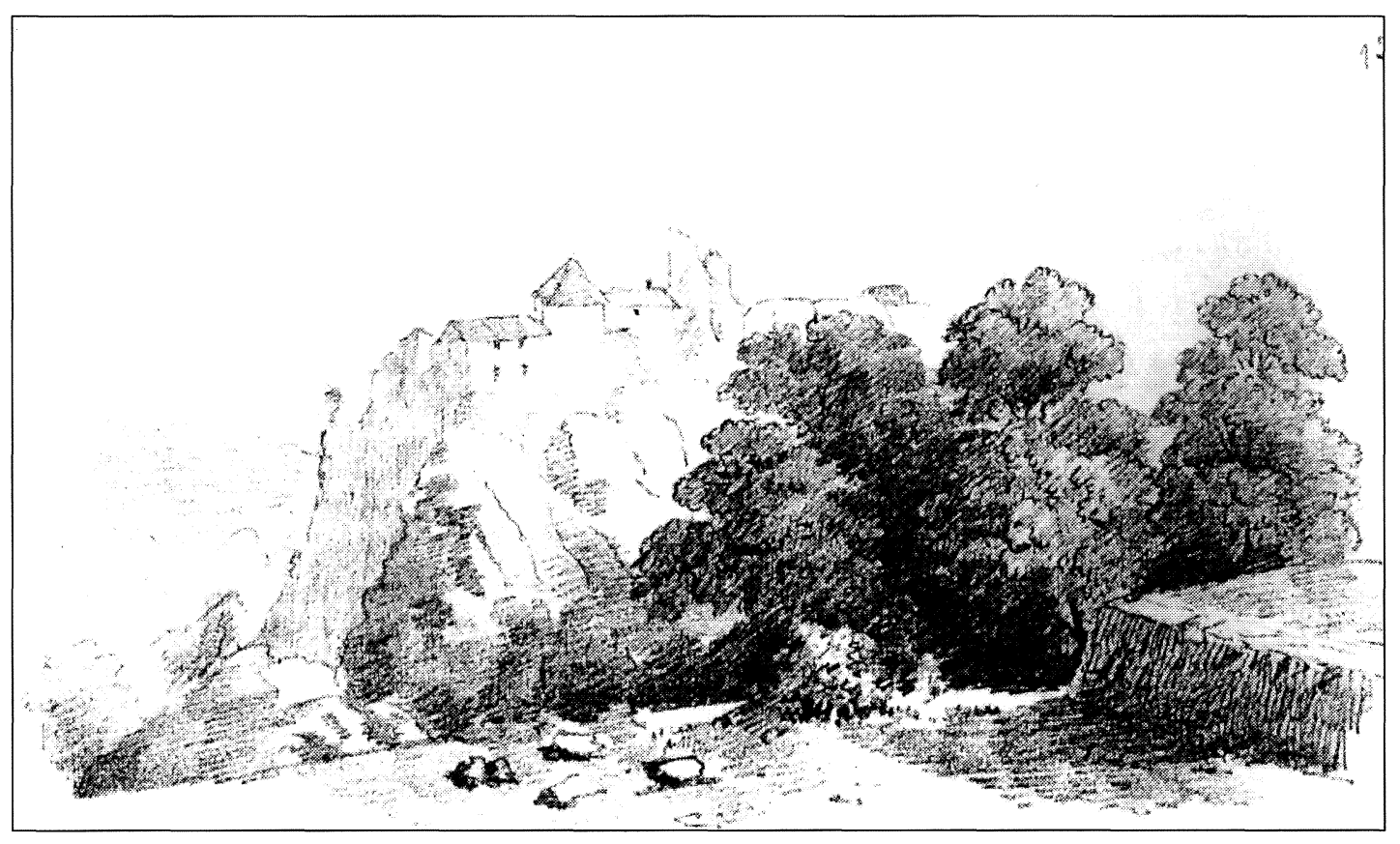

19

20

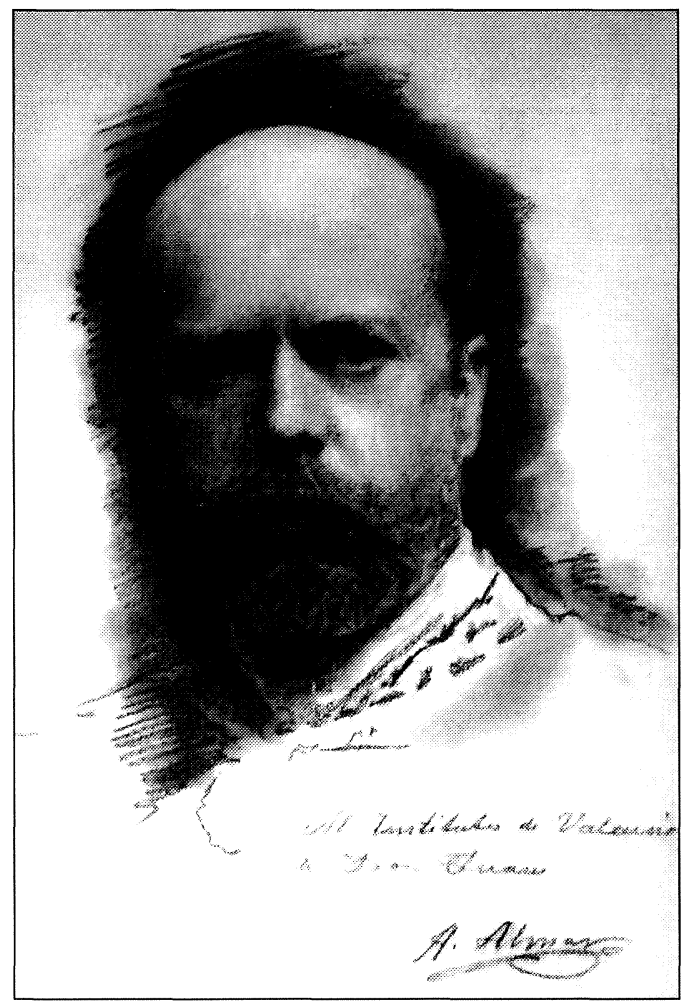

21

Fig. 19. Anónimo, Paisaje.

Fig. 20. A. Almar, Retrato de Guillermo J. de Osma.

Fig. 21. ¿Antonia de Bañuelos y Tourdike?, Retrato de dama. 
guna mural y de temas costumbrista moralizante. Se trata de un espléndido dibujo ejecutado al carbón, con ágiles trazos y acusado sombreado, que titulamos Mujer sentada pensativa, temática que acostumbró a representar referida a damas llenas de ausencia, serenidad y melancolía, que se atempera con unos aislados toques de color ${ }^{31}$.

Nuevamente aparece retratado Guillermo de Osma en un aterciopelado dibujo realizado al carbón por Agustín Almar ${ }^{32}$, que se había formado en la segunda mitad del siglo xix en la valenciana Academia de Bellas Artes de San Carlos, pintor de asuntos costumbristas y ceramista, en función de cuya actividad debió trabar relación con el retratado, que era experto coleccionista en la materia, como lo prueba la dedicatoria que figura al pie ${ }^{33}$.

Restan por citar dos piezas que podrían datarse a fines del xIx o principios del xx. La primera es un expresivo y atractivo dibujo al carbón ${ }^{34}$, de correctísima ejecución plástica, al estilo de Ramón Casas (1866-1932), que refleja la serenidad y clase de la Dama retratada, cuya firma, de trazo poco claro, aunque parece A. de Baviu, ofrece dudas a la hora de hacer una atribución segura. No obstante, por las características plásticas de la obra, podría ser Antonia de Bañuelos y Tourdike, pintora de la segunda mitad del siglo xix, formada en Roma y París, especialista en retratos, que participó en las Exposiciones de Bellas Artes (1878 y 1880) y en la Universal (1889), ambas de París, así como en las Nacionales de 1887 y 1890 en las que recibió mención honorífica y segunda medalla, respectivamente ${ }^{35}$. El segundo, y último, es un anónimo Paisaje, ejecutado con soltura en el uso del lápiz mediante un procedimiento simple, consistente en trazos paralelos degradados con los cuales se consigue la volumetría de las formas, y que posiblemente formó parte del típico cuaderno de apuntes de campo, a tenor del número que figura en uno de sus extremos ${ }^{36}$.

José Antonio Ocaña Martínez

C.S.I.C.

\section{DOCUMENTOS INÉDITOS PARA LA BIOGRAFÍA DE LOS MACIP *}

La importante exposición sobre Vicente Macip realizada en el Museo de Bellas Artes de Valencia en 1997, acompañada del extenso catálogo ' con textos de su comisario el doctor Fernando Benito y de José Luis Galdón, ha supuesto, desde su rigurosa tarea de investigación, la

${ }^{31} \mathrm{~N}^{\circ}$ inv. $^{\circ} \mathrm{I}-7869,457 \times 524 \mathrm{~mm}$, lápiz carbón con toques de cera azul y ocre sobre papel. Fd. ${ }^{\circ}$ a.s.d. «Juan Llimona».

32 VV. AA, Cien años de pintura en España y Portugal, 1830-1930, Anticuaria, op. cit., vol. I, pp. 130-131; Garín Ortiz, F., Catálogo-Guía del Museo de Bellas Artes de San Carlos de Valencia, Inst. Alfonso el Magnánimo, Valencia, 1955, p. 207; Tormo, E., Valencia, los museos de Valencia, Valencia, 1932, p. 73.

${ }_{33}$ N. ${ }^{\circ}$ inv. I-6215, 460 x 294 mm, carbón sobre papel Sucesores de Torras. En a.i.d. «A. Almar. Al Instituto de Valencia de Don Juan». No debe descartarse la posibilidad de que este retrato haya sido realizado a partir de una de las abundantes fotografías que se conservan de G. de Osma, muchas de ellas en el Instituto, lo que no resta calidad alguna al dibujo.

${ }^{34} \mathrm{~N}$. $^{\circ}$ inv. $^{\circ} \mathrm{I}-6311,320 \times 230 \mathrm{~mm}$, carbón sobre papel y ligera aguada. En a.i.d. «¿A de Bañuelos»?

35 Osorio y Bernard, M., Galería Bibliográfica de Artistas españoles del s. XIX, op. cit, p. 67; Pantorba, B. de, Historia y crítica de las exposiciones nacionales de Bellas Artes, Madrid, 1980, op. cit, p. 373 y VV. AA, Cien años de pintura en España y Portugal, 1830-1930, Anticuaria, op. cit. Vol. I, p. 262.

${ }_{36} \mathrm{~N}^{\circ}$ inv. $^{\circ} \mathrm{I}-7867,161 \times 272 \mathrm{~mm}$, lápiz sobre papel. En a.s.d. «12» a lápiz.

* Agradezco a M. José López Azorín, la fundamental aportación documental para la elaboración de este artículo, y a Eva M. ${ }^{a}$ García Barambio la transcripción de gran parte de estos documentos. Queremos señalar que este artículo se redactó con anterioridad a la exposición Joan de Joanes. Una nueva visión del artista y su obra inaugurada en enero de 2000 en el Museo de Bellas Artes de Valencia. Consideramos entonces, al saber que se estaba preparando esta muestra, que era oportuno entregar a sus responsables una copia del artículo, apareciendo debidamente citado en su catálogo como «en publicación» - pues había sido ya aceptada su publicación en esta revista- De este catálogo extrae algún dato L. Hernández Guardiola para un artículo sobre Jerónimo de Córdoba, citando también adecuadamente la fuente.

1 Vicente Macip (h.1475-1550). Museo de Bellas Artes de Valencia, febrero-abril de 1997. 